\title{
A Year in Lockdown:
}

\section{How the Waves of COVID-19 Impact Internet Traffic}

By Anja Feldmann, Oliver Gasser, Franziska Lichtblau, Enric Pujol, Ingmar Poese, Christoph Dietzel, Daniel Wagner, Matthias Wichtlhuber, Juan Tapiador, Narseo Vallina-Rodriguez, Oliver Hohlfeld, and Georgios Smaragdakis

\begin{abstract}
In March 2020, the World Health Organization declared the Corona Virus 2019 (COVID-19) outbreak a global pandemic. As a result, billions of people were either encouraged or forced by their governments to stay home to reduce the spread of the virus. This caused many to turn to the Internet for work, education, social interaction, and entertainment. With the Internet demand rising at an unprecedented rate, the question of whether the Internet could sustain this additional load emerged. To answer this question, this paper will review the impact of the first year of the COVID-19 pandemic on Internet traffic in order to analyze its performance. In order to keep our study broad, we collect and analyze Internet traffic data from multiple locations at the core and edge of the Internet. From this, we characterize how traffic and application demands change, to describe the "new normal," and explain how the Internet reacted during these unprecedented times.
\end{abstract}

\section{INTRODUCTION}

The worldwide pandemic caused by the Corona Virus 2019 (COVID-19) is a once-in-a-generation global phenomenon that changed the lives of billions of people and destabilized the interconnected world economy. What started as a local health emergency in Asia at the end of 2019, turned into a global event at the beginning of 2020 when the first cases appeared on other continents. By March 2020, the World Health Organization (WHO) declared COVID-19 as a pandemic, causing many governments around the globe to impose strict lockdowns of economic and social activities to reduce the spread of COVID-19. These measures changed the habits of a large fraction of the global population, who now depend on residential Internet connectivity for work, education, social interaction, and entertainment.

Changes in Internet user behavior are common, but they normally occur gradually and over long periods of time. Notable examples of such changes are the increase in demand for peer-to-peer applications that happened in the early 2000s; the increase of traffic served by content delivery networks-such as an increase in streamingthat took place in the 2010s; and, more recently, the elevated demand for mobile applications. In all of these cases, the telecommunications industry and network operator community reacted by increasing the investment on network infrastructure. However, the changes in
Internet user behavior during the pandemic have been unique because the shifts took place within weeks, leaving. hardly any time to react. This raised questions of whether user behavior changes yield to changes in Internet traffic and, more importantly, concerns if the Internet is able to sustain this additional load.

In this paper, we investigate the impact of the COVID19 pandemic on the Internet traffic by analyzing more than two years of Internet traffic data including the first year of the pandemic. More specifically, we characterize the overall traffic shifts and the changes in demand for particular applications that became very popular in a short amount of time. During the process, we try to understand if there is a "new normal" in Internet traffic and to see how the Internet reacted in these unprecedented times. We summarize our observations for the spring 2020 wave (February 2020 to June 2020) and then extend our study for the fall ${ }^{2} 2020$ wave (September 2020 to February 2021). To that end, we collect and analyze network traffic data from multiple vantage points, such as a large Internet Service Provider (ISP) in Europe, three Internet Exchange Points (IXPs) in Europe and the US, as well as a mobile operator and a metropolitan academic network in Europe (REDIMadrid).

Our main observations can be summarized as follows:

- Changes in traffic volume follow demand changes, causing a traffic surge of $15-20 \%$ during the fall 2020 lockdown for the ISP/IXPs in our study. In summer 2020 , after the reopening of the economy, an increase of about $20 \%$ at one IXP, but only $6 \%$ at the Tier- 1 ISP, is still visible. The fall 2020 wave also had an impact, with the annual traffic increase in 2020 being higher than in a typical year.

- The observed traffic increase mostly takes place during nontraditional peak hours. Daily traffic patterns are moving to weekend-like patterns, especially during the spring 2020 lockdown.

a We use "spring" and "fall" from the viewpoint of the Northern hemisphere, where our vantage points are located. Exchange both terms for the Southern hemisphere.

An earlier version of this paper was published in the Proceedings of the $20^{\text {th }}$ ACM Internet Measurement Conference (IMC'20). ${ }^{9}$ 
- Traffic related to remote working applications, such as VPN connectivity applications and video-conferencing applications, surges by more than $200 \%$. VPN traffic seems to remain at elevated levels even during the fall 2020 wave.

- Traffic changes across networks differ. For example, in the REDIMadrid campus network, there was a significant drop (by up to 55\%) in traffic volume on workdays after the spring 2020 lockdown as most people were not on campus, but an increase during the fall 2020 lockdown. Traffic at the IXP and the ISP also varies depending on the mandated lockdown policy and due to the different customer profiles.

\section{DATASETS}

The Internet is formed by a network of networks. Depending on their size and position, networks may act as large traffic hubs forwarding network traffic between up to hundreds or even thousands of other networks (backbone/core). In other cases, they are interconnected weakly and closer to the consumer at the edge of the topology.

To obtain a broad perspective on the impact of the pandemic on the Internet, we observe the Internet from multiple and diverse vantage points. They are located at the backbone and peering points of a major Tier-1 Internet Service Provider (ISP), at the core of the Internet-namely three Internet Exchange Points (IXPs) around the globe-and at the edge (a metropolitan university network and a mobile operator).

ISP. A large Central European ISP that provides service to more than 15 million fixed line subscribers and also operates a transit network (Tier-1).

IXPs. An IXP is an interconnection facility where networks become members to exchange traffic with other members across the IXP's infrastructure. In our study, we consider three major IXPs. The largest IXP, namely IXP-CE, is located in Central Europe. It has more than 900 members and a peak traffic of more than 9 Tbps. The second IXP, namely IXP-SE, is located in Southern Europe and has more than 170 members. The third IXP, namely IXP-US, is located at the US East Coast and has more than 250 members.
REDIMadrid university network. We collect and analyze data from the REDIMadrid academic network, which interconnects 16 independent universities and research centers in the region of Madrid. It serves nearly 290,000 users such as students, faculty, researchers, student halls, WiFi networks (such as eduroam), and administrative and support staff.

Mobile operator: A European mobile provider with more than 40 million customers.

At each vantage point, we collect and analyze traffic flows before and during the pandemic. This allows us to reason about the impact of the COVID-19 pandemic on Internet traffic and discuss related traffic shifts. In order to guarantee user privacy, we only analyze anonymized or aggregated datasets. For additional details on our measurement methodology, we refer to Feldmann et al. ${ }^{9}$

\section{NETWORK TRAFFIC SHIFTS}

To understand traffic changes during the COVID-19 pandemic, we first look for overall changes in well-established traffic patterns before, during, and after the strictest lockdown periods for both the spring and fall 2020 waves. Because all data sources exhibit very different traffic characteristics and volumes, we normalize the data to make it comparable. In Figure 1, we show the normalized aggregated traffic of the ISP, IXPs, and mobile operator vantage points from January 2019 until the end of February 2021. We normalize this by using the traffic of the first week of January 2020 for each corresponding vantage point.

\subsection{Macroscopic observations}

In Figure 1, we annotate the week of the initial lockdowns in the countries that host the ISP and IXPs in Central and Southern Europe, and the mobile operator. Although the exact dates of when the lockdown was imposed differ across Europe, these dates are very close to each other and followed the declaration of COVID-19 as a pandemic by the WHO. A first observation is that the ISP and IXPs in Central and Southern Europe show a more than 20\% traffic increase within a week after the official announcement of the

Figure 1. Traffic changes during the COVID-19 pandemic's spring and fall waves at our Internet vantage points.

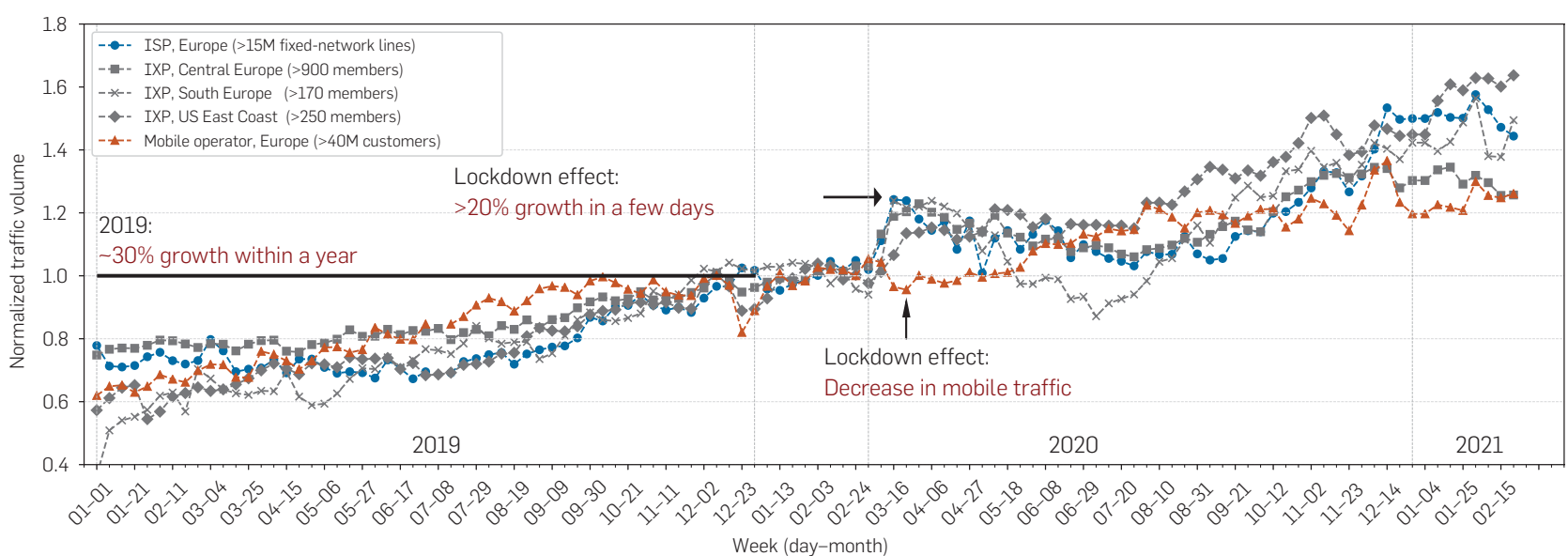


lockdown. This could be perceived as a "moderate" surge in traffic. However, in Internet reality, this is a substantial increase in traffic in only a short period of time. To put it into perspective, the figure shows that the annual increase of 2019 was around $30 \%$, which is similar to the annual increase in previous years. This means that the expected traffic increase in one year happened only within a couple of weeks in March 2020 following the spring 2020 lockdown. Well-provisioned networks, such as the ones we measured for our study, could cope with this surge. However, networks that "ran hot" may have faced problems as this increase is significant and takes place in a relatively short period of time. In sharp contrast, the traffic of the European mobile operator decreased as users switched to WiFi and reduced their commute and traveling. This aligns with reports in other studies. ${ }^{15}$ It is worth noting that, during the same period, the traffic at the IXP at the U.S. East Coast surged only by $2 \%$ as there was no announcement of strict lockdowns in the U.S. at that time.

The impact of the spring wave was significant as the traffic levels remain at same elevated level during the lockdown. The traffic of the IXP at the U.S. East Coast increased significantly when lockdown measures took place by the state authorities. However, when the economy opened again after June 2020, we observe a slight decrease of ISP and IXPs traffic as well as an increase of the mobile operator's traffic.

The impact of the fall wave is clearly visible in traffic patterns beginning in September 2020. The traffic at the ISP and all IXPs surged again, whereas the traffic of the mobile operator declined, except for the holiday period at the end of 2020. Although the lockdowns in the fall of 2020 differ significantly from country to country, and in some cases there were lockdowns with on-off periods, the impact of the fall wave was significant. The 2020 annual increase for the ISP and the IXPs varied between $35 \%$ and $50 \%$, that is, higher than the expected annual increase. The mobile operator showed an annual increase of around $20 \%$, which is lower than expected. As the fall wave of COVID-19 continues into 2021, we observe similar trends until February 2021. It is also worth noting that in some countries, the fall wave was a superposition of multiple waves of COVID-19 and its mutations, which were faced with harder lockdown restrictions. Additionally, the severe weather conditions in Southern Europe with historic snow volumes in January and February 2021 may have also played an additional role in keeping people at home and the corresponding increase of Internet traffic at the ISP and IXPs.

Figure 2 focuses on the ISP, where we show the normalized aggregated traffic for each month for the years 20182021. Although during the years 2018-2019 the traffic increase was around $30 \%$ compared to the same month in the previous year, there was a dramatic change after March 2020. To understand this, we annotate the increase between a given month and the one in the previous year for 2019, 2020 until February 2021 above the bars in the figure. During the spring 2020 wave, the traffic in each month increased by around $45 \%$ compared to the traffic in 2019; the peak was in April 2020 with about 50\%. Between August and October 2020, the traffic increase was similar to

\section{Figure 2. ISP monthly normalized downstream traffic change during the COVID-19 pandemic with percentage increase compared to the} previous year.

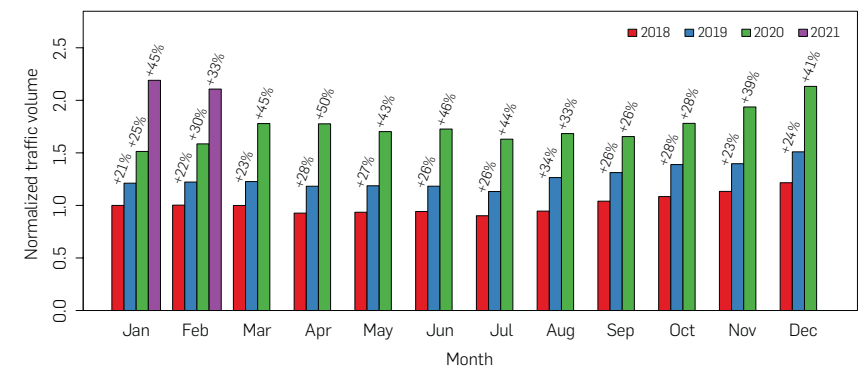

previous years. This aligns well with good weather conditions and the relaxation of lockdown policies. However, this period was followed by stronger lockdown policies, and there is again a surge that continues until the end of our observation period, that is, February 2021.

The traffic increases we have seen across vantage points can arise unexpectedly and may create a need for capacity increases by network operators. We observed capacity increases in the order of 1,500 Gbps (3\%) across many IXP members at the IXP-CE alone. Beyond our datasets, some networks publicly reported that traffic shifts due to the pandemic resulted in partial connectivity issues and required new interconnections. ${ }^{8}{ }^{20}$ The vantage points in this paper range from extremely large to moderate sizes with sufficient resources and a lot of experience in network provisioning and resilience. In general, smaller networks with limited resources may not be able to plan with sufficient spare capacities and fast enough reaction times to compensate for such sudden changes in demand. In fact, performance degradation issues have been reported in less developed regions, ${ }^{1}$ which also highlights the digital divide.

\subsection{Drastic shifts in Internet usage patterns}

Beyond the macroscopic observations, our analysis sheds light on the shifts in Internet usage patterns that are also relevant to network operation and management. The Internet's regular workday traffic patterns are significantly different from weekend patterns. ${ }^{13}$ On workdays, traffic peaks are concentrated in the evening, typically between 18:00 and midnight, also referred to as "peak hours." During the weekend, the activity is more distributed also in the nonpeak hours as more people are at home and using the Internet.

With the pandemic lockdown in March, this workday traffic pattern shifts toward a continuous weekend-like pattern. More specifically, we call a traffic pattern a workday pattern if the traffic spikes in the evening hours and a weekend pattern if its main activity gains significant momentum from approximately 9:00 to 10:00 am. Figure $3 \mathrm{a}$ and $\mathrm{b}$ shows the normalized traffic for days classified as weekend-like on the top and for workday-like on the bottom. If the classification is in line with the actual day (workday or weekend) the bars are colored in blue, otherwise they are colored in orange. We find that up to mid-March, most days are classified correctly. The only exception is the holiday period at the 
Figure 3. Drastic shifts in Internet usage patterns during the COVID-19 pandemic. Classification of weekend- and workday-like patterns.

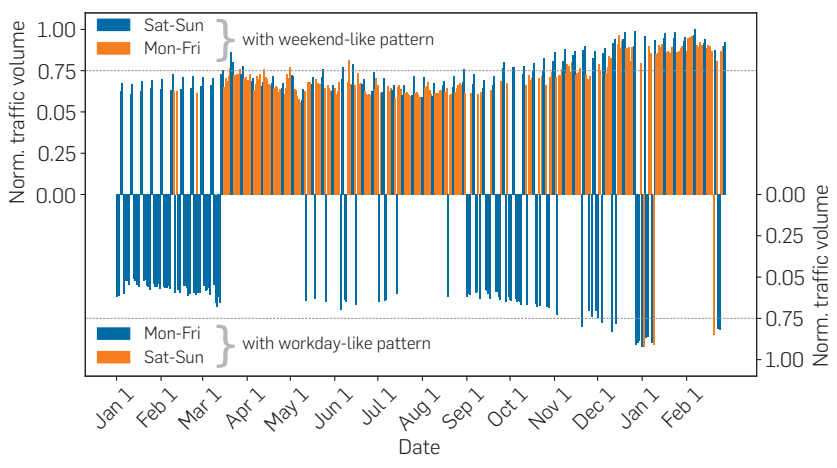

(a)ISP-CE:Weekend-like(top)versus workday-like(bottom).

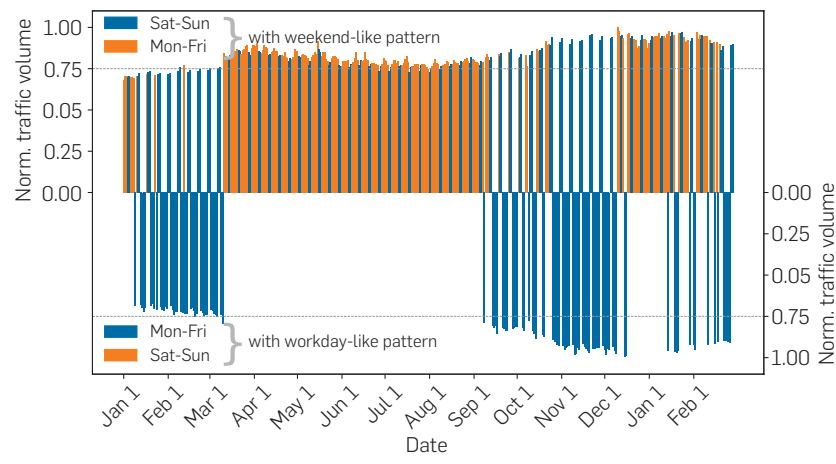

(b)IXP-CE:Weekend-like(top)versus Workday-like(bottom). beginning of the year in Figure $3 \mathrm{~b}$. This pattern changes drastically once the lockdown measures are implemented. Indeed, almost all days are classified as weekend-like. This change persists in Figure $3 \mathrm{~b}$ until the end of August due to the vacation period, which is consistent with the behavior observed in 2019 (not shown). By contrast, Figure 3a shows that the shift toward a weekend-like pattern becomes less dominant as countermeasures were relaxed in mid-May, but in August, the pattern resembles again the weekend-pattern due to the vacation period.

During the period of August 2020-December 2020, the patterns both at the ISP and the IXP are back to the usual weekday and weekend pattern. When the first lockdown of the fall COVID-19 wave was imposed in December 2020, this pattern was disrupted, more noticeably at the IXP. In the first two months of 2021, there was a mixed pattern for both the ISP and the IXP. We conclude that we still observe a transient behavior in 2021 and it is unclear whether the changes of daily usage patterns are here to stay.

\subsection{Effect on the traffic asymmetry}

As we discussed in the previous sections, residential traffic surged both during the spring and fall COVID-19 waves. In this section, we take a closer look on the directionality of the traffic and comment on new patterns in upstream and downstream traffic. Recall that residential traffic is asymmetric in nature, that is, downstream traffic is typically many times higher than the upstream one. This is to be expected as users send less traffic than they receive when using applications such as video streaming and browsing. In Figure 4 (top), we show the aggregated upstream traffic from October 2019 to end of February 2021. There is a slight increase in upstream traffic after the first lockdown in mid-March 2020. This trend manifests itself in the following months: The minimum, but more noticeably the maximum upstream level increase across the rest of the observation period.

As a result of the general elevated traffic levels, the downstream traffic also increases during this period. To assess if there is a change in the established ratio between upstream and downstream traffic, in Figure 4 (bottom), we plot the ratio of upstream versus downstream traffic. Before the
Figure 4. ISP aggregated over 8 hours traffic during October 2019February 2021: upstream traffic normalized growth (top), and downstream versus upstream traffic ratio (bottom).

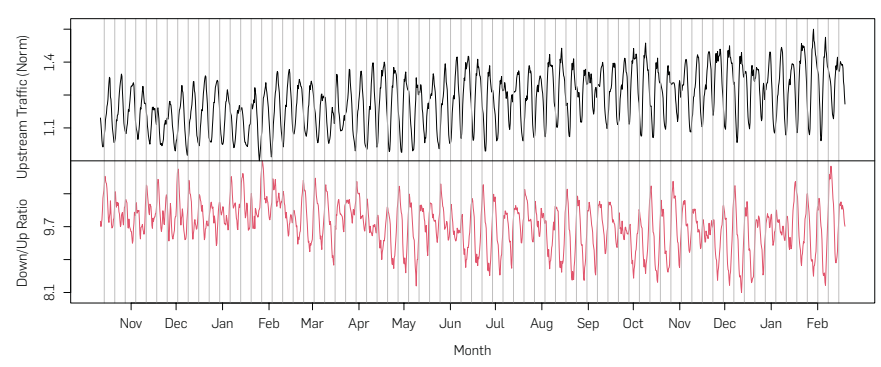

COVID-19 pandemic, typical values of this ratio were around 9.8 with some noticeable variation. After the initial lockdown in the beginning of March 2020 and until the end of February 2021, this pattern changes. Indeed, the ratio of upstream versus downstream traffic decreases significantly, with typical values around 9 and very high variation. During the weekdays, this ratio is as low as 8.1. This shows that the relative increase of the upstream traffic is up to $18 \%$ higher than that of the increase of the downstream traffic. An independent study that analyzes traffic data from U.S. ISPs reported even higher upstream versus downstream traffic ratios during the pandemic. ${ }^{2}$ We attribute this to the increase in remote working and teleconferencing applications that utilize user upstream bandwidth much more than other popular user applications, for example, video streaming and browsing. This is an important observation as ISPs in general allocate way less upstream than downstream capacity to end users. If we see a persistent change in demand from end users to push more traffic toward the Internet, ISPs may need to adapt their handling of subscriber lines. This is a notable result, because last mile capacity is notoriously expensive for ISPs and hard to replace with new technology.

\section{APPLICATION TRAFFIC SHIFTS}

We now turn our attention toward the traffic shifts for different application classes that were expected to be affected by 
the COVID-19 pandemic, namely Web conferencing applications, Video-on-Demand streaming, online gaming, and traffic that originates from university service networks. We refer to Feldmann et al. ${ }^{9}$ for technical details on how we classified traffic in any of these categories.

\subsection{Application classes' traffic shift}

In Figure 5, we visualize two weeks in the Spring and Fall waves, namely the second week in March 2020, June 2020, December 2020, and January 2021, as the difference of the respective week. We compare them to a base week before the initial lockdowns began, that is, February 20-26, 2020. As the traffic classes we are considering show growth way beyond the expected natural increase over one year, we do not factor out that increase. Each column represents one hour of a day. This approach enables quick visual identification of increased/ decreased application class usage compared to pre-COVID-19 times. We focus on the observations gathered at the ISP and the IXP in Central Europe (IXP-CE) vantage points.

Web conferencing. Web conferencing applications have seen a dramatic surge during the lockdown periods. In this category, the ISP and IXP-CE experience a large traffic growth in March-right after the first lockdown beganspanning across all hours of the day, especially during weekdays. This trend accelerates in June and culminates in December and January, with an increase exceeding 300\% compared to the base week at both vantage points. Notably, in December and January, the extreme growth also persists at weekends. This indicates that not only work life has moved online but private social activities did as well.

Video-on-demand. Video streaming applications' usage shows high growth both in the Spring and Fall waves. Interestingly, the ISP only sees a moderate growth during the lockdown in the first half of March followed by a reduction of volume in the second half of March below the pre-COVID-19 reference time frame. We attribute this to major streaming companies reducing their streaming resolution in Europe by mid-March for 30 days. ${ }^{18}$ In the case of the IXP, a similar but not that much pronounced trend can be observed in March. However, there is a significant increase of the traffic related to Video-on-Demand in June, December and January, which exceeds $200 \%$ (IXP) and $100 \%$ (ISP) for some days, especially on weekends indicating that more people stayed at home during leisure time instead of going outside.

Gaming. The strong growth of gaming applications is more coherent at the IXP vantage point, especially during the day. Although the ISP shows a significant increase during morning hours, it generally leans toward declining in the Spring wave. Note that this effect is mainly caused by unusually high traffic levels in this category during our baseline week in February 2020. The initial download of a game nowadays supersedes the amount of data transferred although playing these high levels may relate to new releases or updates of popular games. Gaming applications, typically used in the evening or at weekends, are now used at any time.

Figure 5. ISP (top), IXP-CE (bottom) heatmaps of application classes' traffic at the ISP, and IXPs during COVID-19 pandemic: spring and fall waves. Each subplot shows the change in the aggregated traffic volume per hour for the respective class compared to the base week in February 2020. White areas mark missing data.
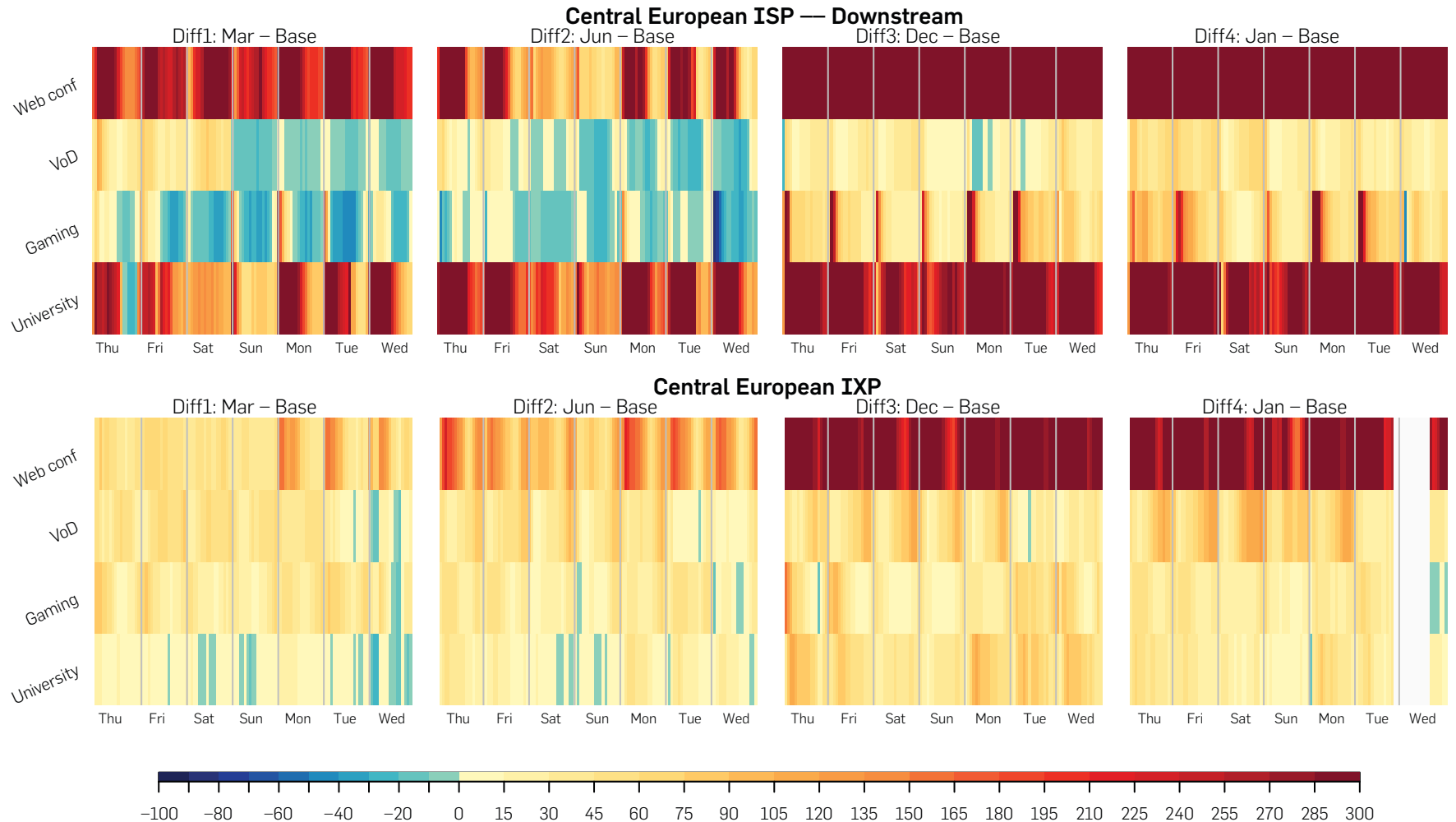
The trend starts to flatten in June- this may in relation with people going on vacation or spending more time outside. The ISP sees an increase up to $300 \%$ in gaming-related traffic during the fall wave across all weekdays, but with emphasis to the first half of the day. A similar pattern unfolds at the IXP, but with smaller increases. One explanation for the strong increase at both vantage points in the morning hours is that schools were closed during the fall wave.

University networks. Traffic that originates from such networks behaves similar at both vantage points with the ISP showing a more pronounced trend. Both vantage points see a high increase in traffic especially during the fall wave with a growth of $100 \%$ and more. This growth could be attributed to some European educational networks providing video conferencing solutions, which are now being used by customers of the ISP/ IXP. In December 2020 and January 2021, most academic collaboration and teaching activities moved to an online setting. This is in line with the smaller surge of activity at weekends.

\subsection{VPN traffic shift}

Working from home leads to a higher demand for Virtual Private Network (VPN) solutions as employees need to access firewall-protected resources hosted in internal company networks. We identify VPN traffic using a novel technique based on transport port data as well as DNS data. ${ }^{9}$ In Figure 6, we show the changes in VPN traffic during the spring and fall COVID-19 waves in 2020. We use five weeks of data from the IXP in Central Europe from February 2020 to January 2021, each in different months, to highlight the differences. February 2020 serves as a baseline, that is, to show the state of VPN traffic before COVID-19 restrictions were enforced.

In March 2020-after the first lockdown restrictions were authorized in Europe-we notice a large increase in VPN traffic during working hours. This growth partially recedes in June 2020 as lockdown restrictions are relaxed again and employees could return to their workplaces. Nevertheless, VPN traffic volume is still over the February 2020 baseline levels. In the Fall wave, VPN traffic increases again but not as high as in March 2020.

We also investigate the share of VPN traffic among the total volume across waves. We find that the VPN traffic share remains stable from February to March 2020. This suggests that overall traffic volumes increase, regardless of the application. However, in June 2020, VPN traffic share increases, whereas overall traffic volume decreases. Toward December 2020 and January 2021, we see a slight decrease in the VPN traffic share as the overall traffic gains traction again after the summer holiday.

In summary, we see that VPN traffic increases during working hours since the first lockdown measures were implemented. The increase is higher in the spring wave than in the fall one. This finding aligns with reports indicating that more people in Central Europe were working from home in the Spring wave compared to the fall wave. ${ }^{23}$

\subsection{A view from REDIMadrid}

In addition to investigating changes at ISPs, IXPs, and mobile networks, we analyze changes at a special type of network: REDIMadrid, a large European academic network. As a large portion of traffic is generated by students and staff being physically on the campus, we expect to see quite drastic changes after lockdown measures are imposed. As a response to the COVID-19 pandemic, the regional government of Madrid announced the closure of the educational system from March 11, 2020 onward for the 2019-2020 academic year. By the end of April, most universities had fully transitioned toward an online-lecturing model. The 20202021 academic year followed a semipresence model, allowing for some lectures to take place on campus.

Traffic volume. Figure 7 shows the normalized traffic volume for six different weeks ranging from February 2020 (one week before announcing that the academic system would be closed down) to January 2021. We observe a significant drop in the traffic volume on working days right after the lockdown, with a maximum decrease of up to $55 \%$ on Tuesday and Wednesday. This is expected because users no longer use the network from within the campus. Traffic on weekends sees increments of up to $14 \%$ on Saturday. Similar to what we see at the IXP-CE and the ISP (cf. Section 3.1), traffic patterns on working days and weekends become more similar in terms of total volume. The traffic volume observed in September and December 2020 reveals an increase with respect to the Spring regime, though the pre-COVID-19 levels are not reached. We observe how traffic on weekends continues to increase, and the difference with work days vanishes even further. We note that the week from January 14, 2021 to January 21, 2021 constitutes an interesting anomaly caused by the Filomena snow storm that brought heavy snow to Madrid for over a week. The effects caused severe mobility limitations for more than two weeks, during which campuses were inaccessible and all lectures were canceled. The effect is noticeable as minimum traffic levels across all categories because the lockdown measures were implemented in February 2020.

Traffic in/out ratio. In the days before the lockdown, incoming traffic was up to $15 \times$ the volume of outgoing traffic during workdays. This ratio dropped to around $2 \times$ or $3 \times$ during lockdown, where traditional weekend versus workday patterns also disappeared. This change of traffic asymmetry might be explained by the nature of remote lecturing and

Figure 6. VPN traffic evolution during the COVID-19 pandemic.
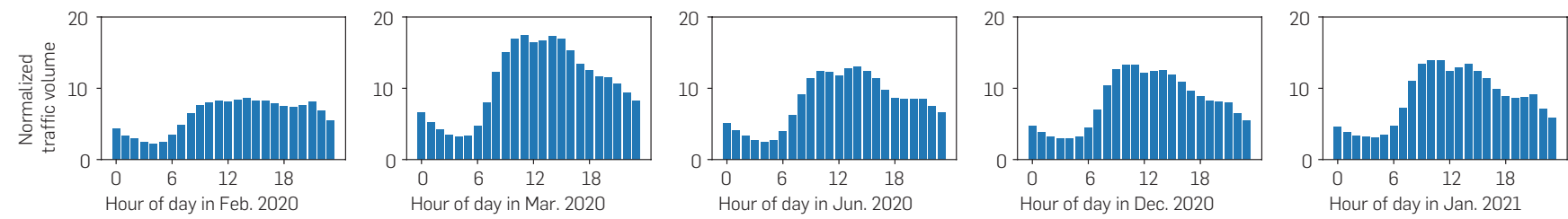


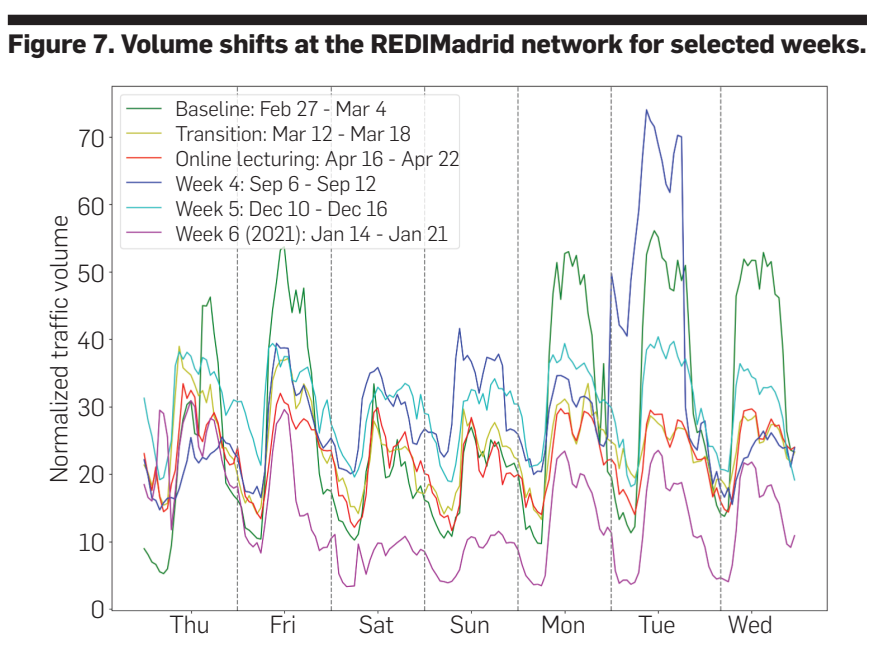

remote working: students and research staff connect to the services hosted at universities to access teaching resources, hence the increase in outgoing traffic. On the other hand, as students and staff no longer access the Internet from the universities, incoming traffic decreases. The semipresence teaching model implemented for the 2020-2021 academic year has resulted in a new intermediary scenario compared to the first COVID-19 wave and the pre-COVID-19 regime. This observation is corroborated at the connection level. Incoming VPN, email, and Web traffic connections remain at high levels compared to February 27,2020 ( $5 \times, 2.3 \times, 2.4 \times$ growth on average, respectively) due to online lecturing and remote working. Outgoing Web traffic and push notification traffic-tightly related to mobile devices - have doubled compared to April 2020 in 2020-2021. However, their overall values are still considerably lower compared to the pre-COVID-19 regime.

\section{DISCUSSION}

Internet operation during the pandemic: A success story. The COVID-19 pandemic “underscored humanity's growing reliance on digital networks for business continuity, employment, education, commerce, banking, healthcare, and a whole host of other essential services." ${ }^{10}$ At the beginning of the pandemic and the first lockdown measures to control its spread, sudden changes in user demand for online services raised concerns for network operators. In fact, the pandemic increased the demand for applications supporting remote teaching and working to guarantee social distancing which manifests itself in our analysis across all vantage points. The Internet could handle this increased load thanks to its original design concept to find efficient routes, ${ }^{22}$ the flexibility and elasticity that cloud services offer, and the increasing connectivity of cloud providers. ${ }^{3,4,12,21,24}$ Our results confirm that most of the applications with the highest absolute and relative increases are cloud-based.

Taming the traffic increase. In this paper, we report a traffic increase of more than $20 \%$ a week after the lockdown began. This is in line with reports of ISPs and $\mathrm{CDNs}^{6,11,16,17}$ as well as IXPs. ${ }^{19}$ Typically, ISPs and CDNs are prepared for a traffic increase of $30 \%$ in a single year period. ${ }^{3,5,14}$ Although networks perform yearly plannings, the pandemic has created substantial shifts within only a few days. As a result, ISPs either needed to benefit from over-provisioned capacity-for example, to handle unexpected traffic spikes such as attacks or flash-crowd events - or add capacity very quickly. The latter was possible due to the adoption of best practices on designing, operating, and provisioning networks which contributed to the smooth transition to the new normal. Due to the advances in network automation and deployment, for example, automated configuration management and robots installing cross connects at IXPs without human involvement, it was possible to cope with the increased demand. For example, DE-CIX, Dubai, managed to quickly enable new ports within a week for Microsoft, which was selected as the country's remote teaching solution for high schools. $^{7}$

\section{CONCLUSION}

Despite the disruption due to COVID-19, life continued thanks to the increased digitization and resilience of our society, with the Internet playing a critical support role for businesses, education, entertainment, purchases, and social interactions. In this paper, we analyze Internet flow data from multiple vantage points in several developed countries. Together, they allow us to gain a good understanding of the impact that the COVID-19 waves and the lockdown measures caused on Internet traffic. One year after the first lockdown measures were enforced, the aggregated traffic volume increased by around $40 \%$, well above the typical expected annual growth. Additionally, workday traffic patterns have rapidly changed and the relative difference to weekend patterns has almost disappeared during lockdowns. Applications for remote working and education, such as VPN and video conferencing, experienced traffic increases beyond $200 \%$.

Our study reveals the importance of covering different lenses to gain a complete picture of these phenomena. Additionally, our observations highlight the importance of approaching traffic engineering with a focus that looks beyond Hypergiant traffic and popular traffic classes to consider "essential" applications for remote working. In fact, our study demonstrates that over-provisioning, proactive network management and automation are key to provide resilient networks that can sustain drastic and unexpected shifts in demand such as those experienced during the COVID-19 pandemic. Yet, as the pandemic is still ongoing, it is critical to continue studying the traffic activity to understand usage shifts during these unprecedented times.

\section{Acknowledgments}

This work has been partially funded by the Federal Ministry of Education and Research of Germany (BMBF, grants BIFOLD 01IS18025A and 01IS18037A, 5G-INSEL 16KIS0691 and AIDOS 16KIS0975K + 16KIS0976), the Spanish Ministry of Science, Innovation and Universities (grants PID2019111429RB-C21 and PID2019-111429RB-C22), and by the Comunidad de Madrid (grants EdgeData-CM P2018/TCS-4499 and CYNAMON-CM P2018/TCS-4566, co-financed by European Structural Funds ESF and FEDER), and by the European Research Council (ERC) Starting Grant ResolutioNet (ERC-StG-679158). The authors would like to acknowledge the 
support offered by David Rincón and César Sánchez (IMDEA Software Institute and REDIMadrid) to access the academic network dataset.

\section{References}

1. Boettger, T., Ibrahim, G., Vallis, B. How the Internet Reacted to Covid-19-A Perspective from Facebook's Edge Network. In ACM IMC (2020).

2. Bronzino, F., Feamster, N., Liu, S., Saxon, J., Schmitt, P. Mapping the Digital Divide: Before, During, and After COVID-19. In TPRC 48 (2021).

3. Labovitz, C. Internet Traffic 2009-2019 (2019). In APRICOT 2019

4. Chiu, Y., Schlinker, B., Radhakrishnan, A.B., Katz-Bassett, E., Govindan, R. Are We One Hop Away from a Better Internet? In SIGCOMM HotNets (2015)

5. Cisco. Cisco Annual Internet Report, 2020. https://www.cisco.com/c/en/us/ solutions/executive-perspectives/ annual-internetreport/index.html.

6. Comcast. COVID-19 network update, 2020. https://corporate.comcast.com/ covid-19/network.

7. DE-CIX. DE-CIX Virtual Get-togetherFocus Middle East \& Asia $22 \mathrm{Apr}$ 2020, 2020. https://www.youtube. $\mathrm{com} /$ watch? $\mathrm{v}=$ DfPt10aopns

8. DFN. German National Research and Education Network: COVID-19

Newsticker, 2020. https://www.dfn.de/ alle-meldungen-aus-demnewstickerzur-covid-19-pandemie/

9. Feldmann, A., Gasser, O., Lichtblau, F. Pujol, E., Poese, I., Dietzel, C., Wagner, D.

Wichtlhuber, M., Tapiador, J.,

Vallina-Rodriguez, N., Hohlfeld, O. Smaragdakis, G. The Lockdown Effect: Implications of the COVID-19 Pandemic on Internet Traffic. In ACM IMC (2020).

10. ITU. Press release: New 'State of
Broadband' report warns of stark inequalities laid bare by COVID-19 crisis (2020). https://www.itu.int/en/ mediacentre/Pages/PR20-2020broadband-commission.aspx.

11. Labovitz, C. Pandemic impact on global Internet traffic. In NANOG 79 (2020).

12. Labovitz, C., Lekel-Johnson, S., McPherson, D., Oberheide, J., Jahanian, F. Internet inter-domain traffic. In ACM SIGCOMM (2010)

13. Lakhina, A., Papagiannaki, K., Crovella, M. Diot, C., Kolaczyk, E.D., Taft, N. Structural analysis of network traffic flows. In ACM SIGMETRICS (2004). with the surge in demand? 2020. https://blogs.akamai.com/2020/04/ can-the-internet-keep-up-with-thesurge-in-demand.html.

15. Lutu, A., Perino, D., Bagnulo, M., characterization of the COVID-19 pandemic impact on a mobile network operator traffic. In ACM IMC (2020).

16. McKeay, M. Parts of a whole: Effect of COVID-19 on US Internet traffic, 2020. https://blogs.akamai.com/ sitr/2020/04/parts-of-a-whole-effect-

17. McKeay, M. The building wave of Internet traffic, 2020. https://blogs. akamai.com/sitr/2020/04/the-buildingwave-of-internet-traffic.html.

18. Netflix. Reducing Netflix traffic where it's needed while maintaining the member experience, 2020. https://media.netflix.com/en/ companyblog/reducing-netflix-
14. Leighton, T. Can the Internet keep up Frias-Martinez, E., Khangosstar, J. A of-covid-19-on-us-internet-traffic.html. traffic-where-its-needed

19. Sanghani, B. COVID-19 \& IXPs. RIPE 80, 2020 https://ripe80.ripe.net/ wp-content/uploads/presentations/ 27-ripe80-covid-ixp-1.pdf.

20. Schilz, B., Maunier, R. Experience on deploying a new remote PoP during COVID-19 restriction. RIPE 80, 2020. https://ripe80.ripe.net/wp-content/ uploads/presentations/26-VolterraRipe-connect-presentation.pdf.

21. Schlinker, B., Kim, H., Cui, T., Katz-Bassett, E., Madhyastha, H.V. Cunha, I., Quinn, J., Hasan, S. Lapukhov, P., Zeng, H. Engineering Egress with edge fabric: Steering oceans of content to the world. In ACM SIGCOMM (2017)

22. Timberg, C. Your Internet is working Thank these Cold War-era pioneers who designed it to handle almost anything. The Washington Post, April 6 2020, 2020. https://www. washingtonpost.com/technology/

Anja Feldmann, Oliver Gasser, and Franziska Lichtblau, Max Planck Institute for Informatics, Saarbrücken, Germany.

Enric Pujol and Ingmar Poese, BENOCS Berlin, Germany.

Christoph Dietzel and Daniel Wagner DE-CIX, Cologne, Germany and Max Planck Institute for Informatics, Saarbrücken, Germany.

Matthias Wichtlhuber, DE-CIX, Cologne, Germany.

(c) This work is licensed under a https://creativecommons.org/licenses/by-nc/4.0/ 2020/04/06/your-internet-is-working thank-these-cold-war-erapioneerswho-designed-it-handle-almostanything/.

23. Wirtschafts- und Sozialwissenschaftliches Institute. Press release Deutlicher Anstieg: 24 Prozent der Erwerbstätigen arbeiten aktuell vorwiegend oder ausschließlich im Homeoffice (in German), 2021 https://www.boeckler.de/pdf/pm wsi 2021 02 16.pdf

24. Yap, K-K., Motiwala, M., Rahe, J., Padgett, S., Holliman, M., Baldus, G. Hines, M., Kim, T., Narayanan, A. Jain, A., Lin, V., Rice, C., Rogan, B., Singh, A., Tanaka, B., Verma, M., Sood, P., Tariq, M., Tierney, M., Trumic, D. Valancius, V., Ying, C., Kallahalla, M., Koley, B., Vahdat, A. Taking the edge off with Espresso: Scale, reliability and programmability for global Internet peering. In ACM SIGCOMM (2017).

Juan Tapiador, Universidad Carlos III de Madrid, Madrid, Spain

Narseo Vallina-Rodriguez, IMDEA Networks, Madrid, Spain and ICSI, Berkeley, USA.

Oliver Hohlfeld, Brandenburg University of Technology, Cottbus, Germany.

Georgios Smaragdakis, TU Berlin, Berlin Institute for the Foundations of Learning and Data, Berlin, Germany and Max Planck Institute for Informatics, Saarbrücken, Germany.

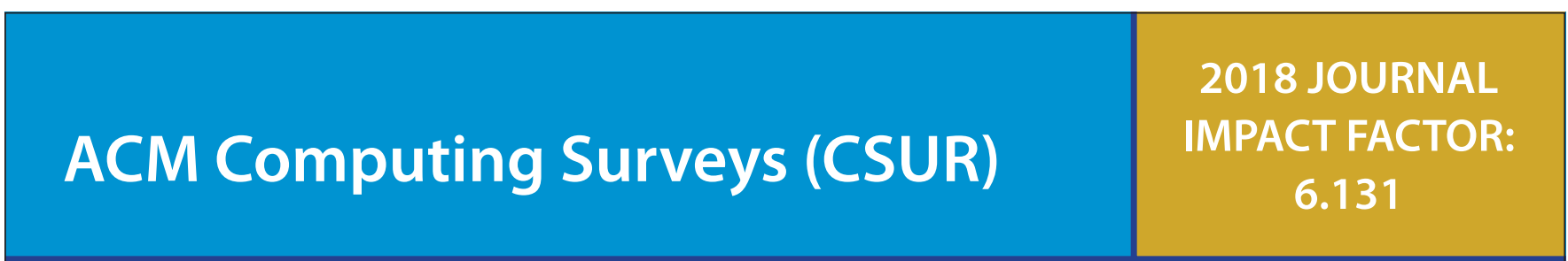

Integration of computer science and engineering knowledge

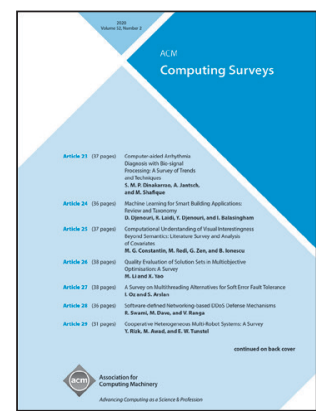

ACM Computing Surveys (CSUR) publishes comprehensive, readable tutorials and survey papers that give guided tours through the literature and explain topics to those who seek to learn the basics of areas outside their specialties. These carefully planned and presented introductions are also an excellent way for professionals to develop perspectives on, and identify trends in, complex technologies. 